\title{
Liderazgo: Pieza clave en el éxito organizacional
}

\author{
Angie Baena Ordosgoitia ${ }^{1}$
}

\begin{abstract}
Resumen
Todas las organizaciones sueñan con encontrar la fórmula secreta que les permita garantizar su permanencia en el mercado y obtener resultados por encima de los esperados. Para lograrlo resulta fundamental contar dentro de las organizaciones con los recursos requeridos, no sólo los económicos y tecnológico, especialmente se debe contar con un recurso humano que cuente con los conocimientos requeridos y que adicionalmente trabaje incansablemente por el cumplimiento de los mismos. Esto sólo se logra cuando se alinean todos con la filosofía organizacional. La manera más sencilla de generarlo es a través del bien ejercido liderazgo. Esta revisión analiza la obra de Nicolás Maquiavelo, enfocada en el ejercicio que como líderes deben hacer las personas que dirigen las organizaciones, sus características, los diferentes estilos y niveles de gerencia basada en liderazgo, el control y seguimiento como pieza clave para la consecución de los objetivos y metas de la compañía, así como la importancia que tienen todos los actores en el cumplimiento de los objetivos.
\end{abstract}

\section{Palabras Claves \\ Liderazgo, estrategia, confianza, organización, éxito}

\begin{abstract}
The common dream on all companies is to find the secret formula that can guarantee them a permanent spot on the market and results above the expected ones. to achieve this is fundamental to have the required resources within the company, not only the economical and technological, specially the human resource needs to be taken into account, this resource needs to have the required knowledge and needs to be committed to reach the objectives on a constant basis. These objectives can only be achieved when all the resources are aligned with the organizational philosophy. The easiest way to reach this result is with a well rounded management. This revision analyzes Niccolò Machiavelli's The Prince, focused on the practice that, as leaders, people that run companies need to have, their characteristics, the different levels and styles of management based on leadership, the tollgates and follow ups as a key piece on target achievement and company goals, as well as the importance that all participants have on the fulfillment of these.
\end{abstract}

\section{Keywords}

Leadership, strategy, trust, organization, success

1. Ingeniería Agroindustrial. Estudiante de la Especialización en Planeación Estratégica y Prospectiva, Universidad Tecnológica de Bolívar. angiebaena16@gmail.com 


\section{Introducción}

El deseo de victoria es un aspecto que caracteriza a la humanidad en general. El poder y la sed de victoria seducen a la mayor parte de la población. En las organizaciones ocurre exactamente lo mismo, todas nacen con el firme propósito de convertirse y posicionarse como la numero uno en el mercado.

Niccolò di Bernardo dei Machiavelli, mejor conocido como Maquiavelo, escribió hace más de 500 años la obra El príncipe. Un manual de gobierno de la época feudal, que ilustra las diferentes situaciones que se pueden enfrentar desde los principados, vistos estos a la luz de las organizaciones como los líderes en los que éstas depositan su confianza para alcanzar el éxito empresarial.

En esta revisión se realiza un análisis del libro El príncipe, a la luz de los elementos de liderazgo, las características propias de los diferentes estilos de gerencia, los niveles que pueden ser alcanzados por los líderes, y los medios para evaluar la eficiencia de las organizaciones. Adicionalmente, se analiza la importancia de los equipos como parte fundamental de las empresas, así como las características que debe tener un buen líder y las ventajas de planear y anticiparse como estrategia fundamental para vencer en la guerra, no por el territorito, sino por la conquista de los clientesg nuevos mercados.

\section{Desarrollo}

La guerra entre naciones fue por muchos años determinante para lograr adquirir nuevos territorios, la lucha incasable por derrotar a quien estaba en el poder y reemplazarlo era casi un arte. Esto lo entendió perfectamente Maquiavelo, quien en el año 1513 escribió una de sus obras más famosas: El príncipe. Dicha obra es el resumen de sus experiencias políticas, en la que se narran los diferentes tipos de principados, cómo estos pueden ser obtenidos y más que eso como pueden ser retenidos bajo una misma persona en el poder (Maquiavelo, 2009). El libro es fácilmente adaptable al mundo de los negocios, en el que los príncipes son esos líderes con los que cuenta o debería contar la organización; los súbditos, soldados e integrantes del ejército son los empleados y/o co- laboradores; y los enemigos son claramente identificables como la competencia.

Para Maquiavelo (2009) administrar, es decir, liderar de forma correcta territorios incluye componentes como la astucia, el pragmatismo, la inteligencia, la firmeza, el manejo de las emociones y el encanto personal; el buen líder debe saber recompensar y castigar a sus súbditos y colaboradores, reconocer los esfuerzos; debe ser prudente, estar siempre alerta, ser flexible al cambio, saber elegir a sus equipos y rodearse de hombres de buen juicio.

El liderazgo es un proceso en el que se influye, guía o dirige a los miembros de un grupo hacia el éxito, buscando la consecución de las metas y de los objetivos organizacionales (Agüera, 2004). Liderar resulta siempre un desafío en la vida empresarial, teniendo en cuenta que para hacerlo se deben considerar los objetivos de la organización, así como los recursos con los que se cuenta (De Oliveira, 2006). Según Maxwell (2007), el liderazgo es un proceso de aprendizaje que se desarrolla a diario, la capacidad de hacerlo determina el nivel de eficiencia que llegue a lograrse en las organizaciones, para esto se requiere adquirir la habilidad de influir sobre los demás, establecer la ruta exacta para lograr los objetivos (estrategia), estar al servicio de los colaboradores, infundir confianza y seguridad en ellos; se debe ser fuerte, utilizar la intuición; saber otorgar poder a otros, enseñar con el ejemplo, buscar siempre el éxito global y no el individual; saber priorizar y tener la capacidad de ceder cuando se requiera.

Un buen líder es aquel capaz de establecer responsabilidades claras, establecer la cantidad correcta de niveles en la estructura de la organización, ubicar a los roles de trabajo en el nivel correcto y cubrirlos con personas capaces de ejercerlos eficazmente, asignar tareas adecuadamente y gerenciar de forma correcta (Lee, 2009).

Un líder es entonces aquel que emplea un conjunto de habilidades para dirigir con éxito la organización. Tal como se aprecia el líder no está relacionado con el jefe, pues este último ocupa un lugar específico en el organigrama y tiene personas a su cargo, pero no implica esto que en la ejecución de su labor logre desarrollar su proceso como lo haría un líder. Se debe recordar que un 


\section{Angie Baena Ordosgoitia}

líder es aquel que tiene la capacidad de aconsejar y guiar, no de ordenar y mandar; inspira entusiasmo en su equipo y no infunde miedo; se preocupa por las personas, su crecimiento y desarrollo; tiene clara la visión de la organización, se gana la confianza de sus colaboradores, trabaja con ellos en equipo, asume riesgos, no le teme al cambio, sabe controlar sus emociones y empodera a su equipo.

La gerencia es una actividad realizada por seres humanos, razón por la cual se encuentra ampliamente influenciada por sus características personales, como elementos determinantes del estilo gerencial (Niebles, Martinez, \& Oñoro, 2007). Éstos pueden ser:

- Autócrata: Establece los objetivos del equipo de trabajo que dirige sin tener en cuenta la opinión, habilidades, experiencias y los conocimientos de ninguno de sus colaboradores; no se comunica; no acepta criticas; y no le interesa retroalimentar al equipo o recibir retroalimentación de estos (Nosnik, 2005).

- Burócrata: Tienen orientación baja hacia las tareas y hacia las relaciones interpersonales cuando estas no son requeridas. Buscan aplicar las reglas y los procedimientos al pie de la letra pero no desarrollan (Crowther, 1993).

- Desarrollista: Dan prioridad al desarrollo personal de los miembros de sus equipos, pero no están orientados al cumplimiento de objetivos (Crowther, 1993).

- Directivo: Proporciona dirección y supervisión clara y específica. Define el papel y ordena a las personas (Arroyo, 2012).

- Coercitivos: Exigen a sus colaboradores el acatamiento inmediato de las órdenes que imponen, son orientados al logro y con buen autocontrol, muy útil en momentos de crisis (Vázquez, 2006).

- Afiliativos: Basan su comportamiento en las personas, muy útiles para resolver problemas y actuar ante situaciones de estrés. (Vázquez, 2006).

- Democráticos: Estos fomentan la comunicación, escuchan, son hábiles para crear con- sensos o para obtener información (Vázquez, 2006).

- Marcapasos: Tienen estándares de rendimiento muy altos, basan su comportamiento en que el colaborador haga lo que el líder hace (Vázquez, 2006).

- Coaching: Son muy útiles para desarrollar a la gente de cara al futuro, con empatía desarrollan fortalezas necesarias para sacar proyectos adelantes, reforzando el clima laboral (Vázquez, 2006).

No existen una fórmula mágica ni una receta secreta que pueda seguirse para convertirse en un líder exitoso y lograr con esto los tan anhelados beneficios para las organizaciones. Sin embargo, lo que sí se puede realizar es definir cuál es el estilo de liderazgo más adecuado para cada empresa y adaptar los perfiles para que sean ocupados con personas con las características requeridas. Estos nuevos cargos eran para Maquiavelo los nuevos principados. Llegar a ellos se lograba a través de la suerte, cometiendo delitos o perversidades, o a través del mérito (Maquiavelo, 2009). Quien llega a un cargo público o privado, o aquel que consigue crecer profesionalmente empleando el engaño, cometiendo delitos y atrocidades tiene garantizado un éxito temporal, corto y efímero. El que lo logra por suerte deberá esforzarse para poder mantenerse en el poder y no tendrá jamás el éxito garantizado. En cambio, el que lo logra por mérito, si bien no tiene el éxito garantizado, sí cuenta con ventajas por encima de los demás; conservarlo dependerá de sus habilidades y destrezas. "Los grandes príncipes por virtud y no por azar, han logrado esto por haber aprovechado la ocasión propicia y darle la forma conveniente. Esta oportunidad sin sus méritos no habría representado nada, así como el mérito sin la oportunidad tampoco hubiera servido" (Maquiavelo, 2009).

Otra de las lecciones que en su obra Maquiavelo (2009) deja de manifiesto es la necesidad de los líderes de conocer la historia de otros, "el príncipe debe leer historia y estudiar las acciones de los grandes hombres para ver cómo se conducían". Esto permite analizar los errores cometidos con anterioridad para no repetirlos y 
conocer los patrones de éxito para replicarlos. El buen líder debe ser analítico, crítico y evaluador. Debe imitar las razones de la victoria de los grandes personajes y evitar los motivos de sus derrotas (Maquiavelo, 2009)

La crueldad y la clemencia son otras de las características de liderazgo definidas por Maquiavelo en su obra. Responder la pregunta ¿es mejor ser temido que amado, o amado que tenido? no es tan sencillo como parece. Él afirma que todos los líderes deben ser tenidos por clementes y no por crueles, sin embargo, deben ser cuidadosos de emplear de manera inadecuada la clemencia. Tampoco se deben preocupar por ser tildados como crueles, siempre y cuando esa crueldad tenga como finalidad mantener unidos y fieles a los súbditos. Para Maquiavelo es mejor ser más temido que amado y más amado que temido, ambas cosas a la vez, pero como es muy difícil tener ambas características al mismo tiempo, afirma que es mejor ser temido. "El amor depende de la voluntad de los hombres y el temor de la voluntad del príncipe, uno prudente debe apoyarse en lo suyo y no en lo ajeno, tratando siempre de evitar el odio" (Maquiavelo, 2009). Más que amor o temor la orientación debe darse al respeto y la admiración. Los colaboradores que respetan a sus líderes los siguen de manera incondicional y buscan alcanzar los mismos objetivos, la admiración acompaña esta premisa. Si bien es cierto que el amor es un vínculo afectivo que al cambiar puede llegar a su extremo opuesto: el odio, y generar traiciones o peligro para los procesos, éste sí puede ser controlado por el líder, al igual que el temor que se infunda en sus colaboradores puede generar miedo al castigo, y ese miedo representar falta de compromiso, dejadez e incluso oportunismo. Para el líder es fundamental evitar ser despreciado y odiado, para esto no debe ser ni despreciable ni odioso; no debe ser voluble, ni frívolo, ni pusilánime o irresoluto; debe demostrar grandeza, valentía, seriedad y fuerza; adquirir la autoridad suficiente para que nadie piense en engañarlo o envolverlo con intrigas; debe cuidarse de las conspiraciones en su contra; debe ser feroz como el león y astuto como el zorro, y emplear ambas habilidades en los momentos adecuados (Maquiavelo, 2009).
Es importante tener en cuenta que el líder no será líder si no tiene a quien liderar. Los colaboradores, el equipo de trabajo, los súbditos, el ejército, las tropas, no importa el nombre que reciban, son pieza clave en el éxito de los procesos. "La primera opinión que se tiene del buen o mal juicio de un príncipe, se funda con base a los hombres que lo rodean. Cuando no elige personas competentes y fieles, no podrá considerarse prudente a un príncipe cuyo principal error lo comete en la elección de las personas que lo rodean (Maquiavelo, 2009).

Un equipo está conformado por recursos, el talento humano es fundamental. Maquiavelo (2009) afirma que las tropas pueden ser propias, mercenarias, auxiliares o mixtas. Las mercenarias son inútiles y peligrosas, éstos son desleales, ambiciosos, valientes frente a los amigos pero cobardes al enfrentarse a los enemigos, traicionan fácilmente a quien los guía. Por su parte los auxiliares nunca le pertenecerán al segundo que las utilice, seguirán siempre las órdenes de quien para ellos es su líder y jamás verán a quien los emplee como su orientador, pues no comparten su visión. Las mejores tropas serán siempre las propias, empleados por los líderes prudentes que prefieren perder con los suyos que vencer con los ajenos, pues esta victoria nunca es verdadera ni duradera. Como el equipo es tan imprescindible para la obtención de resultados y para el desarrollo real del liderazgo, uno de los puntos clave a tener en cuenta está relacionado con las recompensas y los castigos. Estos deben ser justos, producto del mérito y orientados siempre a fortalecer actitudes positivas o corregir las negativas.

Según Maxwell (2012) existen cinco niveles de liderazgo. El primero es considerado el nivel de los derechos, la gente lo sigue porque consideran, tienen que hacerlo, teniendo en cuenta su posición; es el muy conocido jefe. El segundo es el nivel de las relaciones, las personas lo siguen porque quieren hacerlo, logra ganarse la confianza de su equipo, su respeto y admiración, por lo que le es fácil influir en ellos. El tercer nivel es el de los resultados, en éste es seguido por sus logros y avances para y como el equipo. El cuarto nivel es considerado de reproducción, la gente lo sigue por lo que ha hecho por ellos, por 


\section{Angie Baena Ordosgoitia}

el apoyo para el desarrollo de sus potenciales, se gana el respeto por las adecuadas intervenciones en su vida personal y laboral. Por último se encuentra el nivel de respeto, la gente lo sigue por lo que es y lo que representa. En éste el líder dedica sus esfuerzos al desarrollo de las personas que hacen parte de su equipo y de la organización en general, por lo que es seguido sin reparos.

La planificación es un aspecto clave "el príncipe nunca debe dejar de lado el estudio de la guerra, en tiempos de paz se debe pensar en ello más que en tiempos de guerra" (Maquiavelo, 2009).

El buen líder debe ser capaz de establecer planes de trabajo, de definir quiénes se encargarán de cada aspecto y de asignar funciones. Es fundamental la construcción de escenarios fatalistas y victoriosos que permitan la construcción de las estrategias que serán implementadas. Sin embargo, todo esto es insuficiente si no se realiza un adecuado seguimiento y control. También es tarea del líder establecer mecanismos que permitan evaluar la situación actual de la organización, saber exactamente cómo van, dónde están los errores o los cuellos de botella, tomar las medidas para corregirlos, y mejor aún para prevenirlos. Con esto se logra incrementar los niveles de eficiencia organizacional.

\section{Conclusiones}

Uno de los retos más grandes que enfrentan las organizaciones es encontrar personal calificado y cualificado, no solamente en el aspecto técnico, sino también en el humano.

Hoy en día las empresas se preocupan por el desarrollo de sus objetivos, pero también por el de las personas que integran la organización, pues son estos los únicos capaces de aportar para lograr el crecimiento, el cumplimiento de los objetivos y el éxito en el mercado. Para esto las empresas requieren disminuir o eliminar el número de jefes, e incrementar en su totalidad los líderes. Personas con altas capacidades, cualidades y habilidades.

Un buen líder es aquel que pone al servicio de la organización sus conocimientos estratégicos, tácticos, operativos y humanos. Es aquel capaz de adaptarse a los cambios, de guiar y orientar a los miembros de su equipo, de infundir en ellos confianza, seguridad y respeto. Liderazgo implica asignación de funciones, distribución de recursos, construcción de estrategias, evaluación y seguimiento de los planes, evolución permanente.

Existen muchos modelos o estilos de liderazgo. Hay líderes burócratas y autócratas, hoy por hoy mandados a recoger. También existen líderes coercitivos que sólo persiguen resultados a cualquier costo, aun cuando este exija pasar por encima de sus colaboradores. Los líderes democráticos son más participativos, construyen espacios de reflexión y fortalecen el trabajo en equipo, sin dejar de perseguir los resultados. Por último, un nuevo modelo es el Coaching, también conocido como líder transformador, traduciendo literalmente entrenador. Éste está orientado especialmente al desarrollo de los colaboradores, y como este desarrollo personal le apunta al crecimiento organizacional a través del logro de objetivos. Las empresas deben tener claridad absoluta de sus procesos, conocer exactamente qué tipo de líder requiere cada uno y perfilarlos de tal forma que puedan ser dirigidos por quienes puedan aportar más.

Para Maquiavelo (2009) el cambio era lo único seguro. Por esto los príncipes, que en el mundo empresarial representan a los líderes, debían estar preparados para afrontarlos con éxito. El secreto era la anticipación, la preparación con antelación al mismo; el conocimiento de la historia, del pasado, para identificar las causas que llevaron a los antecesores a cosechar éxitos y afrontar fracasos, con la finalidad de replicar los primeros y no repetir los segundos. La adaptación siempre será más fácil con el conocimiento del enemigo, es decir, de la competencia, y por supuesto del conocimiento de uno mismo, es decir, de la organización. La planificación y la preparación constante también son fundamentales a la hora de atacar un territorio (nuevos mercados).

Uno de los puntos más complejos es quizás la administración adecuada el personal. Rodearse de las personas adecuadas, es fundamental para obtener éxito en las empresas.

Aun cuando la postura de Maquiavelo es en algunas ocasiones tosca, dura y fuerte. El secreto está 


\section{Liderazgo: Pieza clave en el éxito organizacional}

en la interpretación y adaptación que se le dé a su escrito. No se debe estar de acuerdo con todo, es claro, sin embargo, sus apuntes son muy útiles y se mantienen vigentes en un mundo que cada vez avanza a mayor ritmo.

\section{Referencias bibliográficas}

Agüera, E. (2004). Liderazgo y compromiso social (1 ${ }^{\text {a }}$ edición ed.). Puebla, México: Benemérita Universidad Autónoma de Puebla. Dirección General de Fomento Editorial.

Crowther, W. (1993). Manual de Investigación-Acción para la evaluación científica en el ámbito administrativo. EUNED.

De Oliveira, P. (2006). Técnicas de liderazgo hoy. Bogotá, Colombia: Editoria Santuario.

Lee, N. (2009). La práctica del liderazgo gerencial. Estados Unidos: Xlibris Corporation.

Maquiavelo, N. (2009). El príncipe (1 ${ }^{\mathrm{a}}$ edición ed.). (A. Lisa, Trad.) Madrid, España: EDAF, S.L.

Maxwell, J. (2007). Las 21 leyes irrefutables del liderazgo. Nashville, Tennessee, Estados Unidos: Grupo Nelson, Inc.

Maxwell, J. (2012). Los 5 niveles de liderazgo. Estados Unidos: Center Street.

Vázquez, R. (2006). Habilidad directivas y técnicas de liderazgo. Su aplicación en la gestión de equipos de trabajo. Vigo, España: Ideaspropias Editorial.

Niebles, E., Martinez, J., \& Oñoro, R. (2007). Procesos Desarrollados Por Gerentes Sociales de Ong 's Exitosas en El Ámbito de la Gestión Del Tercer Sector en Cartagena. Cartagena, Colombia: EUMED.

Nosnik, A. (2005). Culturas organizacionales: origen, consolidación y desarrollo. España: Netbiblo, S.L. 